\title{
EchoGéo
}

$47 \mid 2019$

Nouvelles géographies de la collecte

\section{Penser la cueillette de plantes médicinales sauvages dans des sociétés en transition : le cas guyanais}

Marc-Alexandre Tareau, Lucie Dejouhanet, Guillaume Odonne, Marianne Palisse et Clarisse Ansoe

\section{(2) OpenEdition}

Journals

Édition électronique

URL : https://journals.openedition.org/echogeo/16679

DOI : 10.4000/echogeo.16679

ISSN : 1963-1197

Éditeur

Pôle de recherche pour l'organisation et la diffusion de l'information géographique (CNRS UMR 8586)

Référence électronique

Marc-Alexandre Tareau, Lucie Dejouhanet, Guillaume Odonne, Marianne Palisse et Clarisse Ansoe, "Penser la cueillette de plantes médicinales sauvages dans des sociétés en transition : le cas guyanais », EchoGéo [En ligne], 47 | 2019, mis en ligne le 21 avril 2019, consulté le 10 août 2021. URL http://journals.openedition.org/echogeo/16679; DOI : https://doi.org/10.4000/echogeo.16679

Ce document a été généré automatiquement le 10 août 2021.

EchoGéo est mis à disposition selon les termes de la licence Creative Commons Attribution - Pas d'Utilisation Commerciale - Pas de Modification 4.0 International (CC BY-NC-ND) 


\title{
Penser la cueillette de plantes médicinales sauvages dans des sociétés en transition : le cas guyanais
}

\author{
Marc-Alexandre Tareau, Lucie Dejouhanet, Guillaume Odonne, Marianne \\ Palisse et Clarisse Ansoe
}

Remerciements à l'association Mama Bobi, pour les conseils et le support à Saint-Laurent du Maroni, et aux habitants de Sosoité, Santi Pasi, Cayenne et à tous ceux qui ont bien voulu nous expliquer le pourquoi de ces brassées de plantes que nous découvrions entre leurs mains aux détours de notre enquête.

Ce travail a été financé en partie par des subventions du Labex CEBA (ANR-10-LABX-25-01, Investissement d'Avenir géré par l'Agence Nationale de la Recherche) et son projet ReKABioS. Il a bénéficié du support du projet Oyamar financé par le Feder Guyane. Il s'inscrit également dans le projet «Les territoires de la ville: de l'archipel des Antilles au plateau des Guyanes : espaces, sociétés et relations (XVI $-X X I^{e}$ s.) » de l'EA 929 AIHP-GEODE, ainsi que dans le travail de thèse de doctorat de Marc-Alexandre Tareau sur les «Phytothérapies métissées du littoral guyanais».

\section{Introduction}

1 « Dans cette forêt, il n'y a rien. Des joggeurs, des prostituées, des gens qui font pisser leurs chiens et peut-être quelques personnes qui ramassent des plantes ». Cette phrase, prononcée par l'un des responsables d'une agence d'urbanisme guyanaise lors de notre enquête, reflète bien ce que représente la cueillette de plantes médicinales en Guyane pour ceux qui n'y sont pas initiés : une pratique mal connue, mésestimée et souvent perçue comme anecdotique voire insignifiante. Pourtant, on peut croiser tous les jours des personnes ramassant des plantes le long des routes du littoral guyanais, autant en milieu rural qu'en milieu urbain, pour satisfaire leurs besoins de phytothérapie ou, dans une moindre mesure, pour les revendre. D'ailleurs, il est possible d'estimer à $60 \%$ 
environ les plantes médicinales consommées par les habitants du littoral guyanais issues de la cueillette (données non publiées, thèse de doctorat en cours de MA Tareau). Mais l'opposition opérée depuis longtemps en Europe entre le sauvage et le domestique (Descola, 2004a) $)^{1}$ à la base des théories évolutionnistes du xix $\mathrm{e}^{\text {siècle }}$ (Morgan, 1877) nous pousse souvent à percevoir la cueillette comme une survivance, sans prendre en compte la complexité et la pluralité des interrelations constantes entretenues entre les sociétés et les végétaux (Garine-Wichatitsky, 1997).

2 C'est pour comprendre les formes d'organisation de ces pratiques négligées que nous avons décidé de les étudier à travers le territoire guyanais, marqué par une multiculturalité, un dynamisme démographique et des flux migratoires importants (Piantoni, 2011). Cet article a pour objet de caractériser ces pratiques de cueillette de plantes médicinales, d'en comprendre la diversité, mais aussi d'en saisir la pertinence pour interroger le fonctionnement de sociétés en transition, marquées par un exode rural important, par une transformation rapide de leurs structures sociales et culturelles et de leur milieu de vie et par la cohabitation de plus en plus prégnante de groupes d'origines différentes ayant des rapports à la nature divers.

Ce travail procède d'une multiplicité de méthodes mises en œuvre sur une période allant de janvier 2016 à avril 2018. Des entretiens semi-directifs ont été menés sur l'île de Cayenne ainsi que dans la région du bas-Maroni - frontalière du Suriname voisin auprès des différentes composantes de la population habitant ces espaces (illustration 1). Des marchands de plantes aromatiques et médicinales (PAM) ont été interrogés, plus de 300 échantillons d'herbier collectés et déposés à l'Herbier de l'IRD à Cayenne (CAY Herbarium) ${ }^{2}$ et une spatialisation de la collecte a pu être effectuée en accompagnant des cueilleurs sur leurs lieux de prélèvement. Pour analyser la place de la cueillette dans une société guyanaise en transition, nous présentons deux cas d'études : la collecte urbaine, dans l'île de Cayenne, par des populations diverses, et la cueillette de plantes médicinales effectuée par les Ndjuka de la région du bas-Maroni. Les activités de collecte de ces populations se répartissent dans l'espace selon une structure en "cercles », dont les spécificités perdurent, sous une forme variable, en ville. Enfin, à partir de la situation guyanaise, nous proposons une conceptualisation des modes de cueillette fondée sur les modalités de leur spatialisation et sur l'introduction des notions "d'hétérogénéité » et de «finalité » comme facteurs explicatifs de ces processus. 
Illustration 1 - Carte de localisation

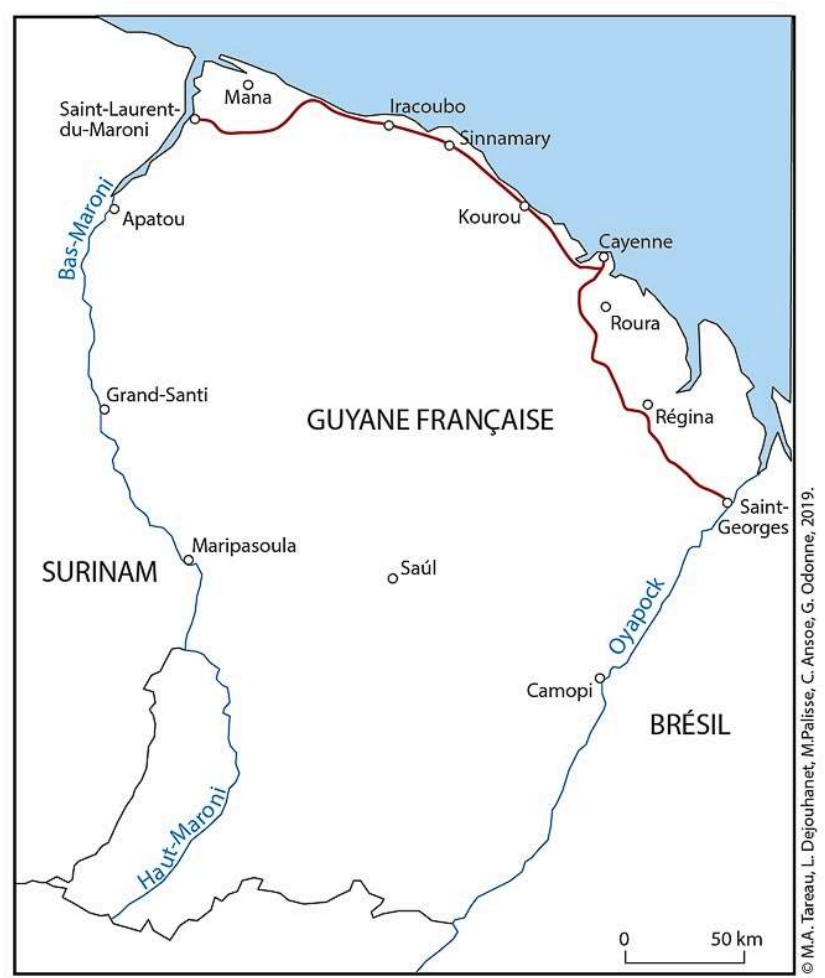

\section{Du village à la ville : la Guyane, une société en transition.}

La Guyane est marquée par l'histoire complexe de son peuplement qui a donné lieu à une forte diversité culturelle. À la suite de la conquête coloniale cohabitent sur ce territoire des populations d'origine européenne, amérindiennes, marronnes (issues de la résistance et de la fuite d'esclaves des plantations du Suriname aux XVII ${ }^{\mathrm{e}}$ et $\mathrm{XVIII}^{\mathrm{e}}$ siècles), et créoles (issues du métissage pendant et après l'esclavage). Ces dernières intègrent à leur mode de vie, dès le $\mathrm{XIX}^{\mathrm{e}}$ siècle, divers apports issus de l'immigration. L'immigration en Guyane est continue mais c'est à partir des années 1970 que les migrations spontanées à destination de la Guyane deviennent vraiment importantes. Les migrants proviennent principalement du Brésil, d'Haïti et du Suriname, mais aussi de divers pays d'Amérique latine et d'Asie (Piantoni, 2011). Dans le même temps, la Guyane est le cadre de divers mouvements de population. Depuis les années 1950, l'administration s'efforce de regrouper et de sédentariser les Amérindiens et Marrons (Guyon, 2011). Un exode rural important se met aussi en place (Jolivet, 1982), en partie lié aux chantiers de la décentralisation et du spatial, et l'on assiste à la croissance spectaculaire des agglomérations de Cayenne, Kourou et de Saint-Laurent du Maroni, cette dernière accueillant de très nombreux réfugiés lors de la guerre civile au Suriname (1986-1992). Ces pôles urbains sont marqués par la présence de populations très diverses, parfois regroupées dans des quartiers en fonction de leur origine, qui conservent souvent des liens avec les territoires dont elles proviennent et qui peuvent circuler entre ces derniers et la ville. Certaines de ces populations ont la citoyenneté française, d'autres sont confrontées aux problèmes des migrants et à la difficulté 
d'obtenir une régularisation administrative. Malgré un relatif cloisonnement, le phénomène urbain donne cependant lieu à des formes d'hybridation entre des populations ayant des modes d'habiter et des rapports au corps et à l'environnement différents. C'est dans ce contexte que vont se développer de nouveaux rapports aux végétaux et émerger des pratiques originales de cueillette.

\section{Un besoin de nature en ville : trouver des plantes utiles à Cayenne.}

Cayenne est le chef-lieu de la Guyane française, sa «capitale » administrative et économique. Avec les communes adjacentes (Matoury et Rémire-Montjoly), elle forme une conurbation dont la population est de 109538 habitants (INSEE, 2014) soit un peu moins de la moitié de la population totale du département. C'est ici que se concentrent l'essentiel des activités et des services administratifs. L'espace résidentiel et les zones d'activités de l'agglomération cayennaise croissent et se densifient sur l'ensemble de la conurbation, bien que quelques îlots de verdure, formés par des "monts $»^{3}$ et des pripri ${ }^{4}$ émaillent le territoire urbain. L'exode rural et les flux migratoires ont amené la population de l'île de Cayenne à augmenter de façon importante ces dernières décennies (2011; Piantoni, 2011). En 2005, 30 \% de la population totale de la Guyane était immigrée ${ }^{5}$ (Hurpeau, 2012), vivant souvent dans les conditions de vie précaires de quartiers spontanés défavorisés. C'est notamment pour répondre à des besoins de santé primaire que les populations urbaines prélèvent dans leur environnement un certain nombre d'espèces médicinales, palliant partiellement les difficultés d'accès à la médecine conventionnelle qu'elles rencontrent. Dans les villes en développement comme Cayenne, des pratiques de phytothérapie et de cueillette issues des cultures d'origine des habitants perdurent aussi (Tareau et al., 2017). Ces usages agissent ainsi comme des marqueurs d'identité culturelle (Volpato et al., 2008) - un véritable « réflexe identitaire et culturel »(Benoît, 2007) -, comme un lien préservé avec les territoires d'origine (Bourdeau-Lepage et Vidal, 2012) et comme une forme d'inscription de ces groupes dans la ville (Roulleau-Berger et Lu, 2003).

6 Les différents groupes résidant à Cayenne se sont approprié l'espace urbain, repérant les endroits où croissent les plantes utiles, profitant de leurs déplacements pour ramasser certaines espèces, dressant des cartes mentales de la ville avec les lieux potentiels de leurs cueillettes. Ainsi, les bordures végétales des routes, les friches urbaines et les « dents creuses » sont des lieux de cueillette privilégiés : ces milieux dits «dégradés", non aménagés, ont une indéniable valeur écologique en termes de diversité et de densité floristique (Sukopp et Hejny, 1990) car ils renferment une végétation spontanée capable de s'adapter aux conditions du milieu urbain (Arnould et al., 2011). Ils sont des « refuges pour la biodiversité » (Clément, 2004). Les parcs urbains et les forêts boisées péri-urbaines sont également des lieux où sont prélevées des espèces médicinales, à l'instar d'ailleurs de ce qui a pu être observé dans d'autres régions du monde (Bussmann et Sharon, 2009 ; Landy et al., 2017 ; Nguenang et al., 2010 ; Wehi P. M. et Wehi W L., 2010). Enfin, les plantes cultivées qui dépassent des jardins urbains et donnent sur la rue font également l'objet d'une pression de cueillette forte de la part des piétons. Toutes ces branches qui dépassent hors des espaces privés deviennent des éléments d'un grand jardin partagé. La ville propose ainsi de multiples opportunités de cueillette, souvent malgré les politiques d'aménagement qui 
voudraient nettoyer la ville de ses friches et limiter la nature aux espaces verts publics ou non (Baudry, 2015).

7 En outre, les villes de Guyane ont, par les spécificités de leur croissance, un paysage particulier. Ces "villes en transition» (Wiel, 1999) se composent de mosaïques de quartiers très différents: quartiers spontanés, habitat planifié, zones humides ou forestières, résidences cossues et bâtiments de l'époque coloniale se côtoient dans l'espace urbain. La planification du développement urbain est souvent en retard par rapport à une urbanisation rapide et mal encadrée. Si les nouveaux quartiers périphériques grignotent l'espace rural autour de la ville, les centres-villes ont cependant tendance à se densifier. Même à Cayenne, le modèle du gradient d'artificialisation des sols allant du centre aux périphéries urbaines (Arnould et Cieslak, 2004) est applicable et entrâne une "collecte en dégradé", diminuant au fur et à mesure de la densification du bâti. Les jardins créoles typiques du vieux centre de Cayenne ont progressivement été occupés par des constructions et la tendance à la "fermeture» de ces espaces végétalisés se poursuit. L'usage d'herbicides et l'«entretien" des bordures de route qui sont régulièrement et de façon assez systématique débroussaillées limitent aussi les possibilités de cueillette dans les centres. Cependant, dans les quartiers populaires de la périphérie, les plantes sauvages utiles sont protégées, comme nous l'indique une habitante haïtienne interrogée :

Anvil yo ka rache se zeb la, yo ka gaze yo, nou nou konnèt yo donk nou ka kite yo (trad. du créole: En ville, ils arrachent ces herbes-là, ils les "gazent », nous, on les connaît donc on les préserve).

8 Enfin, les différents groupes culturels qui habitent Cayenne donnent lieu à une superposition de pratiques de cueillettes variant en fonction des spécificités socioculturelles de ces groupes. Ainsi, les Créoles guyanais ont une connaissance forte des plantes et une mobilité importante, du fait d'un niveau de vie relativement élevé ; par contre, ils ont un besoin relativement faible des plantes pour se soigner, ayant accès à toutes les structures de soin du système de santé français. La cueillette est alors plutôt pratiquée pour le confort et les désordres du quotidien. À l'opposé, les Haïtiens en situation irrégulière ont un besoin important en plantes sauvages, du fait de leur accès limité aux structures hospitalières; par contre, ils ont une mobilité restreinte et doivent donc se contenter des plantes disponibles dans leur quartier ou à proximité. Les plantes sauvages guyanaises ne sont pas forcément connues des Haïtiens qui privilégient les plantes cultivées dont ils peuvent ramener des graines ou qui trouvent des espèces locales similaires à celles qu'ils connaissent. Certaines plantes sauvages bien identifiées sont aussi utilisées: c'est le cas d'asosi ${ }^{6}$, Momordica charantia, cucurbitacée pantropicale d'origine indienne qui pousse spontanément sur les clôtures ou dans les friches (illustration 2). La consommation d'asosi est très répandue chez les Haïtiens, qui font macérer dans du rhum ses parties aériennes. S'il existe une variété cultivée pour ses légumes, c'est la variété sauvage, dont les feuilles sont amères, qui est utilisée de façon exclusive pour la préparation de la boisson éponyme ${ }^{7}$. Les lianes sont donc protégées par les Haïtiens et leur présence est devenue un marqueur végétal des quartiers haïtiens :

Asosi se on bagay ki leve tousèl, nou pa plante'l. Alò se pousa lè nou wè'y nou ka kite'y pou nou pe sa trape lè nou bizwen prepare boutey asosi yo ("L'asosi c'est une plante qui pousse toute seule, on ne la plante pas. C'est pourquoi lorsqu'on la voit on la préserve pour en avoir ensuite pour confectionner les bouteilles d'asosi. »). 
Ces phénomènes de différenciation des pratiques culturelles et leur distribution spatiale ne sont pas sans rappeler les théories d'«écologie urbaine » développées par les sociologues et géographes de l'Ecole de Chicago (Grafmeyer et Joseph, 1984) qui, déjà dans les années 1920 et 1930, montraient comment les néo-migrants se répartissaient dans l'agglomération en fonction d'un processus d'agrégation-ségrégation fondée sur la diversité de leurs origines socio-culturelles et le maintien de comportements et de pratiques culturels spécifiques (Thomas et Znaniecki, 1918). Les importants apports migratoires et ruraux à Cayenne mettent ainsi en relation dans un même lieu des groupes de cultures différentes et ayant des rapports à l'environnement et à la santé divers, souvent marqués par des relations fortes avec les non-humains qui perdurent avec une grande vigueur même dans un contexte peu propice. Cependant, à l'image de la trajectoire de vie du «paysan polonais» de Thomas et Znaniecki (1918) - qui oscille entre " conformisme » à la tradition et tendance au changement -, on peut supposer que cette étape transitionnelle actuelle donnera lieu soit à un effacement progressif de certaines pratiques (Marc, 2007) soit à l'implantation de nouveaux usages ethnobotaniques dans le paysage urbain comme l'ont constaté plusieurs auteurs (Baudry, 2015 ; Baudry et al., 2014 ; Ceuterick et al., 2008 ; Ellena et al., 2012 ; Ososki et al., 2007 ; Pellegrini et Baudry, 2014 ; Tareau et al., 2017 ; van Andel et Carvalheiro, 2013; van Andel et Westers, 2010).

Si la ville change d'aspect par les usages de ses habitants qui contribuent à transformer le paysage des quartiers en cultivant ou en protégeant un certain nombre d'espèces spécifiques, il semble intéressant de se pencher également sur un autre exemple pour saisir la réalité de la cueillette guyanaise aujourd'hui : celui des Ndjuka, grands cueilleurs et utilisateurs de plantes sauvages.

Illustration 2 - Amas spontané de Momordica charantia L. dans une friche urbaine

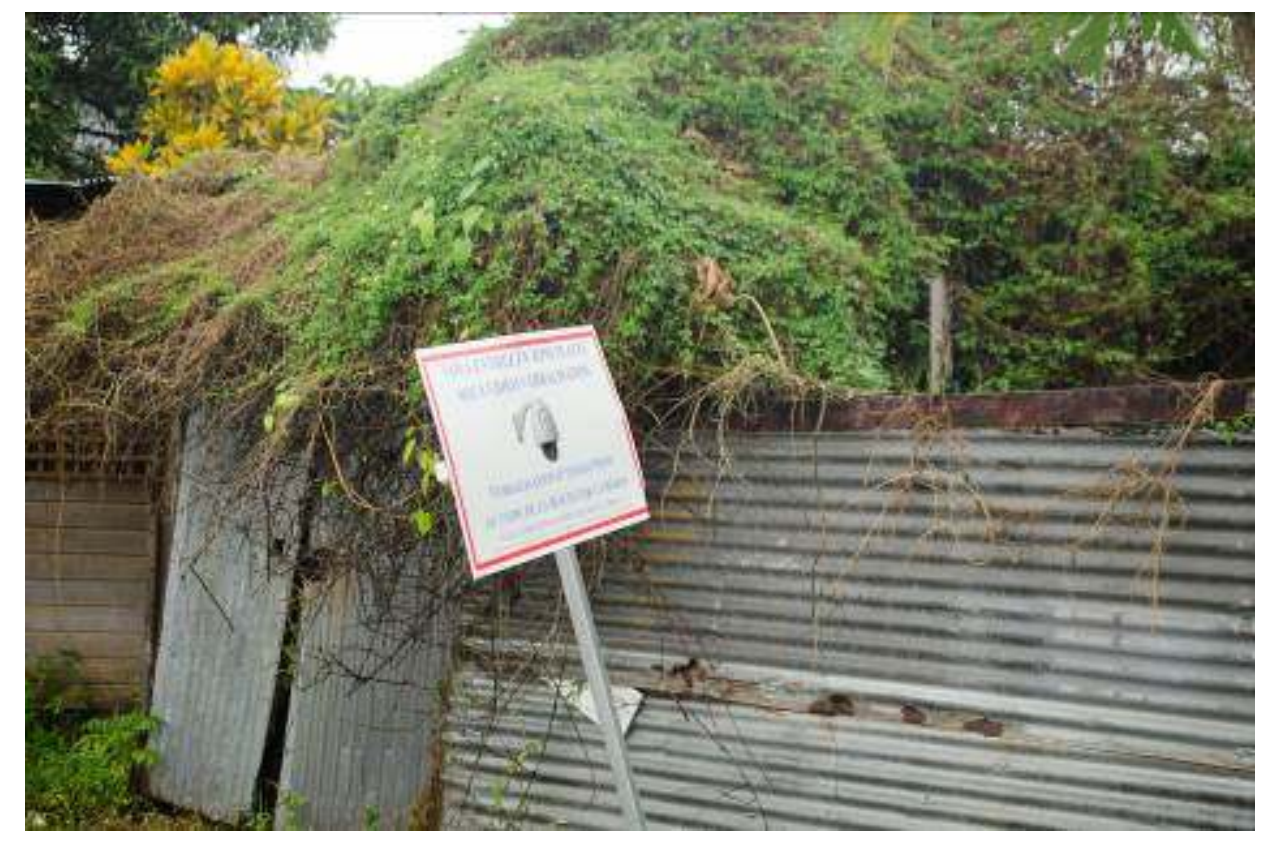

Amas situé à l'entrée de Cayenne, route de Montabo.

Auteur: M.-A. Tareau, mai 2018. 


\section{Un paradigme afro-amazonien de la cueillette : les sociétés marronnes de Guyane.}

11 Les Ndjuka font partie des Noirs-Marrons, appelés Businenge en Guyane, dont les communautés ont pour origine la fuite des esclaves des plantations hollandaises du Suriname, aux XVII ${ }^{e}$ et XVIII ${ }^{e}$ siècles (Price and Price, 2008). Elles se sont très vite constituées en groupes culturels autonomes et singuliers, vivant essentiellement dans l'intérieur forestier (Moomou, 2002). Leur population totale est aujourd'hui estimée à plus de 117600 personnes (Price, 2013b) réparties entre les deux pays frontaliers. Quatre groupes sont présents en Guyane : les Saamaka, les Aluku, les Paamaka, et les Ndjuka. Des milliers de ces derniers, victimes d'un ethnocide durant la guerre civile au Suriname entre 1986 et 1992, ont été forcés de fuir et de s'installer dans l'ouest guyanais (Bourgarel, 1990). La ville de Saint-Laurent du Maroni est aujourd'hui majoritairement habitée par des Businenge (Léobal, 2013). Plusieurs villages situés sur les berges du fleuve Maroni ou répartis dans les savanes ouvertes de la commune côtière de Mana où les camps de réfugiés avaient été installés, accueillent également une population ndjuka importante, qui continue à entretenir des liens forts avec ses villages d'origine en s'y rendant régulièrement (Collomb et Jolivet, 2008).

L'ethnogenèse des groupes marrons, marquée par la fuite face aux milices des colons, les a familiarisés avec les espèces alimentaires et médicinales nécessaires à leur survie en forêt. L'isolement forestier des communautés d'origine (Price, 2013a, 2010; Price et Mintz, 2013), les héritages bio-culturels africains (Carney et Rosomoff, 2011 ; Fleury, 2015, 1991 ; Grimé, 1979 ; van Andel, 2016 ; van Andel et al., 2012 ; Van Andel et al., 2014 ; Vossen et al., 2014) et surtout les nombreux apports amérindiens ${ }^{8}$ ont contribué à façonner une ethnomédecine ndjuka complexe (Sauvain et al., 1988; Vernon, 1993, 1992) dans laquelle interviennent de nombreuses espèces sauvages de collecte (Sauvain et al., 1988). La nécessité quotidienne pour les femmes de prendre des bains de plantes décoctées9 (Fleury, 1996 ; van Andel et al., 2008 ; van't Klooster et al., 2018) et le besoin des hommes de consommer couramment des bita (mélanges de plantes macérées dans du rhum) comme toniques amers pour "se nettoyer le sang» (Sauvain et al., 1988; Tareau et al., 2017 ; van Andel et al., 2012 ; Odonne et al., 2007 ; Sauvain et al., 1988) sont largement permis par les collectes. Enfin, les plantes utilisées dans le cadre des rituels médico-magiques du winti $i^{10}$ sont généralement collectées en milieu sauvage afin de leur assurer une force thérapeutico-spirituelle (kaakiti) plus élevée. En effet, ampuku, esprit de la forêt d'origine africaine (Wooding, 1979) qui symbolise la nature non impactée par l'homme (van Velzen and van Wetering, 1988) et constitue la classe spirituelle la plus convoquée, vit dans les plantes sauvages qui ne doivent donc pas être domestiquées ${ }^{11}$ pour ne pas perdre leur substance sacrée ${ }^{12}$ ou de peur de perturber l'environnement domestique avec la présence trop proches d'esprits craints. À travers le kaakiti qu'ils puisent dans les différentes préparations dans lesquelles interviennent des plantes sauvages, les Marrons font corps avec la forêt, ils deviennent véritablement businenge ("Hommes de la forêt »), ce qui confère à cette thérapeutique fondée sur la cueillette un caractère indissociable de l'identité culturelle des peuples Noirs-Marrons. Cette ontologie animiste au sens de Ph. Descola (2001) est donc caractérisée par la croyance en un panthéon d'entités non-humaines animées qui « habitent » la forêt ${ }^{13}$ et la formule de L. Cabrera (Cabrera, 2003) pour décrire la forêt afro-cubaine d'un point de vue émique pourrait très bien être utilisée à l'égard de la représentation ndjuka de la forêt : 
«Le Noir [...] qui pénètre en plein cœur de la forêt est conscient du contact direct qu'il établit avec les forces naturelles qui l'entourent et qui règnent là sur un domaine qui est le leur. [...] "La forêt est sacrée" parce que les divinités résident et vivent en son sein. Les saints sont dans la forêt plutôt qu'au ciel. » (Cabrera, 2003)

S. Bahuchet (1997), en comparant notamment les Pygmées à certains groupes amazoniens, a montré que deux des caractéristiques principales des populations forestières sont qu'elles puisent une grande partie des ressources végétales dont elles font usage dans la collecte sauvage et que « la forêt vierge est le domaine des esprits ». Les schèmes de représentations symboliques des Noirs-Marrons ndjuka et leurs besoins phyto-médicinaux en espèces sauvages font d'eux d'importants cueilleurs «par culture ». L'une des formules employées au sein de la communauté pour exprimer cette relation intime avec leur milieu végétal environnant tient en : Mi no sabi fu wiri, den sabi fu mi ! («Je ne connais pas les plantes, ce sont elles qui me connaissent »). Pour toutes ces raisons, l'installation en zone urbaine des Ndjuka s'accompagne dans une certaine mesure d'une continuité de la cueillette dans la ville (illustration 3 ). Des filières informelles et plus ou moins organisées d'envoi de plantes de collecte se sont également mises en place afin de répondre aux besoins des "néo-urbains " et des populations émigrées jusqu'en Europe (Van Andel et al., 2007). Des plantes peuvent être achetées au marché. Enfin, lors de leurs allers-retours dans les villages, les populations rurales ramènent régulièrement des plantes cueillies pour leurs propres besoins et approvisionner leur cercle de relations.

Illustration 3 - Femme businenge cueillant des plantes « de maternité »

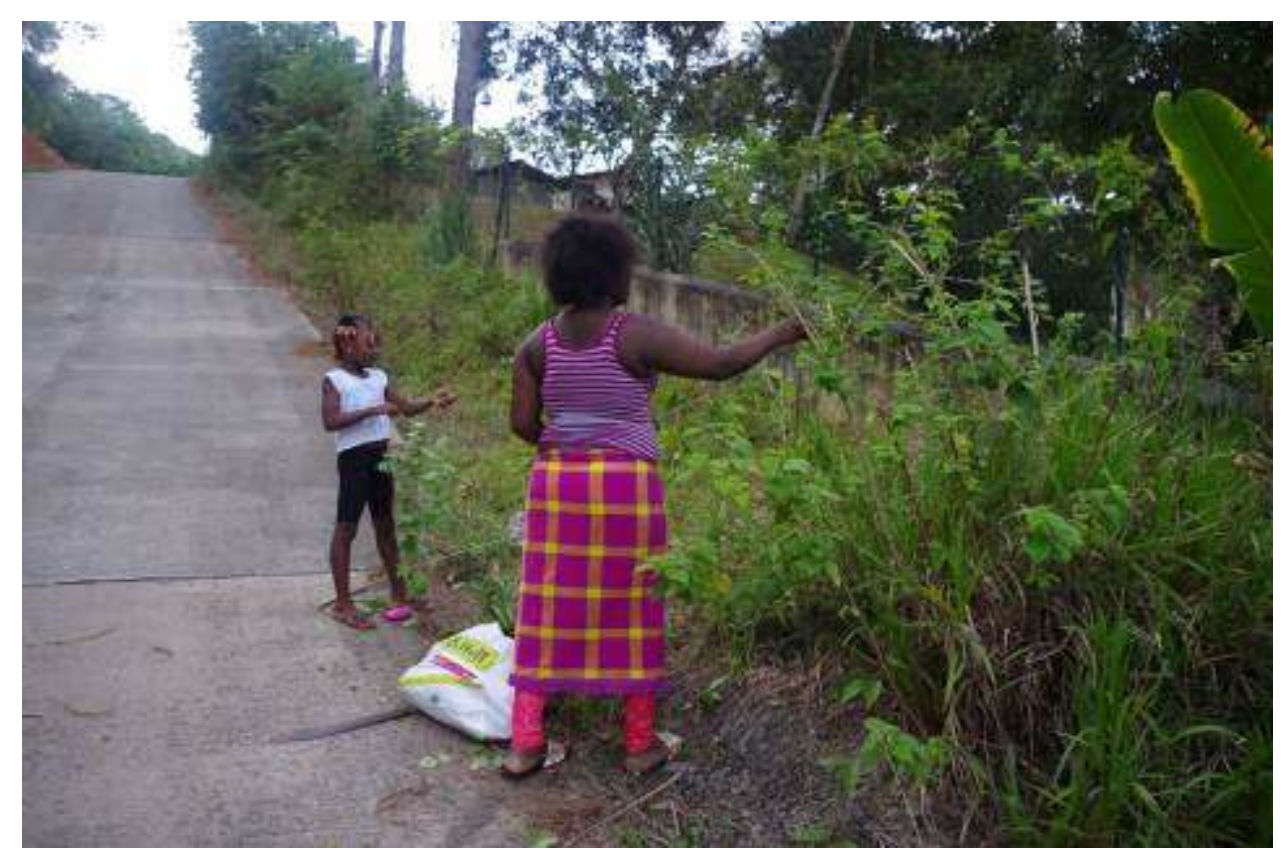

Femme habitant l'île de Cayenne, ici à Rémire-Montjoly.

Auteur : M.-A. Tareau, avril 2018. 


\title{
Les espaces de la cueillette : une géographie culturelle des plantes cueillies
}

\author{
Des espaces de collecte bien différenciés : les « cercles de \\ cueillette » des villages ndjuka
}

L'omniprésence du végétal dans les pratiques quotidiennes des Ndjuka du bas-Maroni entraîne une organisation de la cueillette structurée selon des critères d'usage, de genre et d'espace. Les villageois distinguent ainsi trois espaces de cueillette (illustration 4), similaires au modèle ethnographique classique maison-jardin-forêt décrit par de nombreux anthropologues en Amazonie, dont Ph. Descola au sujet des Achuar ${ }^{14}$ (2004b) ou encore par M. Lenaerts au sujet des Ashéninka (Lenaerts, 2006) ${ }^{15}$. Ce découpage en cercles de l'espace social, avec des représentations symboliques et des usages bien définis inhérents à chacun d'entre eux, a également été décrit en Afrique sub-saharienne (Bahuchet, 1997; Garine-Wichatitsy, 1997). Bien que critiqués avec pertinence par B. Albert et F. M. Le Tourneau, ces espaces correspondent chez les Ndjuka à des pratiques distinctes, nous le verrons plus loin., Toutefois, la proposition d'espaces de collecte réticulaires faite par ces auteurs est également pertinente. Dans le cas Ndjuka, les cercles se caractérisent notamment par une différence relative des espèces qui y sont cueillies - et des usages associés - ainsi que par une répartition genrée de leur utilisation ${ }^{16}$, qui transparaissent clairement dans les discours et confirment le découpage suivant :

\section{L'espace domestique}

Le cercle proximal, autour de la maison, est celui des osu deesi (" remèdes de maison »), c'est-à-dire l'espace où sont protégées les espèces utilisées pour les soins thérapeutiques domestiques, pour soigner les petits maux du quotidien. Il s'agit d'un espace de cueillette essentiellement féminin (Voeks, 2007) : c'est là que les mères viennent chercher un certain nombre de plantes dont elles ont besoin pour soigner leurs enfants- essentiellement des petites herbacées sub-spontanées. La cueillette se fait donc extemporanément, en fonction des besoins. Chez les Ndjuka, les plantes qui poussent autour des maisons sont considérées comme étant en libre accès pour les personnes en ayant besoin. Ceci crée parfois des incompréhensions entre les communautés des différents quartiers, les Ndjuka pouvant cueillir des plantes près des habitations amérindiennes, là où elles sont choyées et plutôt considérées comme des entités privées ${ }^{17}$. 


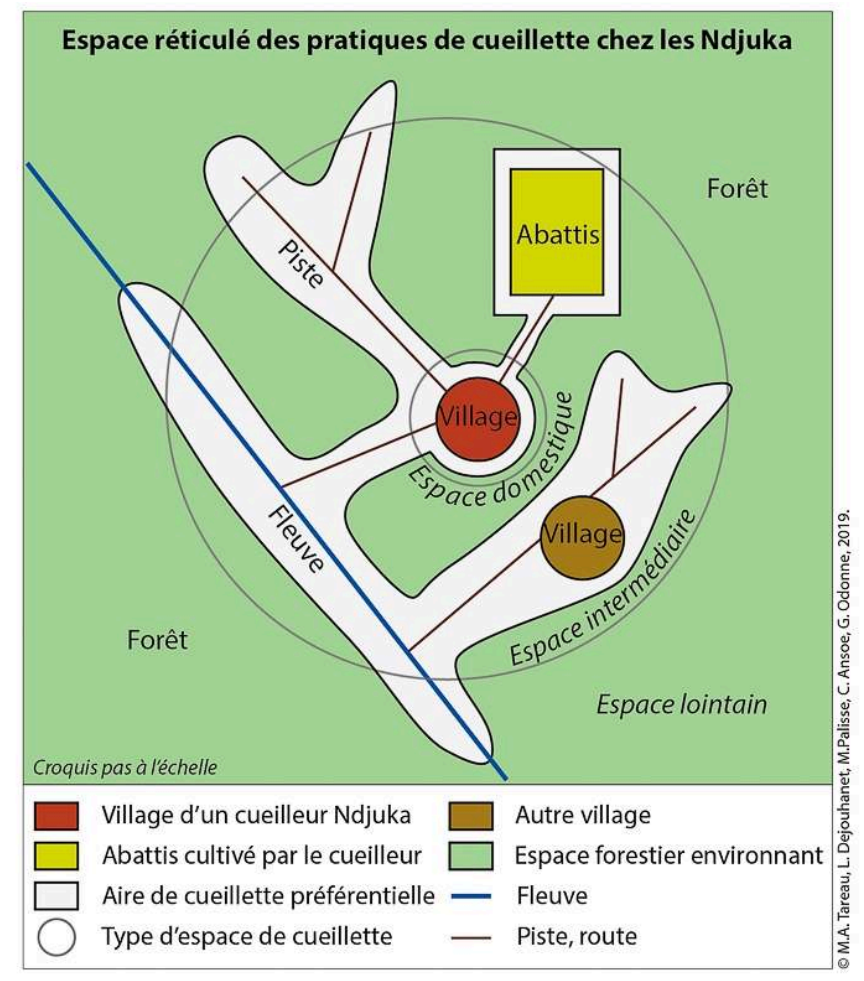

\section{L'espace intermédiaire}

Dans l'espace intermédiaire se font les déplacements quotidiens pour aller à l'abattis ou se rendre dans des lieux voisins du village. Il est donc composé des champs, des pistes et des friches, savanes et forêts secondaires qui les bordent, ainsi que des bords de fleuves et de route. Cet espace peut être désigné comme celui des uman sama deesi, c'està-dire celui des « remèdes pour les femmes ». C'est principalement là en effet que sont récoltées les plantes servant à la toilette des femmes ou pour la maternité, comme l'indique également Havinga (2006) dans son étude de la collecte de plantes chez les Marrons du Suriname. Malgré cela, il s'agit d'un cercle de cueillette mixte : en allant à l'abattis (lieu essentiellement féminin), les femmes ramènent des espèces rudérales ${ }^{18}$ trouvées le long des chemins ou des adventices qui poussent spontanément dans les champs; mais lorsqu'elles ne peuvent pas se déplacer (durant la grossesse ou parce qu'elles sont malades) ou qu'elles ne peuvent pas aller chercher les plantes elles-mêmes à cause de leurs menstruations ${ }^{19}$, ce sont les hommes qui vont chercher pour elles les plantes dont elles ont besoin pour leurs bains. Aussi, lorsque ces derniers empruntent les pistes pour se rendre à la chasse ou dans d'autres villages, ils ramènent également régulièrement des plantes pour leurs épouses. La mobilité et les déplacements sont ici des occasions, voire des moteurs, de collecte, qui font de ce cercle intermédiaire un espace réticulaire, s'allongeant le long des pistes, des routes et des cours d'eau, qui sont autant d'axes d'accès à la ressource, similaire à celui décrit chez les Yanomami.

Cet espace intermédiaire ${ }^{20}$ est sans doute le lieu le plus important de cueillette dans les villages ndjuka. Selon Havinga, $85 \%$ de plantes sauvages collectées par les Marrons du Suriname le sont dans les zones habitées et les forets secondaires (Havinga, 2006). À l'image de ce qui a pu être observé chez les Gimbe et les Duupa du Cameroun (Garine- 
Wichatitsky, 1997), les plantes collectées dans ce cercle sont considérées comme étant les meilleures, dans la mesure où elles constituent un « juste milieu » entre un cercle domestique potentiellement pollué (notamment, ici, par le sang menstruel) et la grande forêt où vivent des "esprits" souvent craints. Les plantes préférées sont donc les rudérales arbustives des bords de piste et les adventices ${ }^{21}$ des champs qui selon certains auteurs peuvent être considérées comme les espèces médianes du continuum entre sauvages et cultivées, des plantes « sauvages domestiquées » en quelque sorte (Harlan et Technique, 1987; Haudricourt et Hédin, 1987). Ces plantes sont certes spontanées mais elles sont épargnées lors du sarclage ou du défrichage voire même encouragées (Harlan et Technique, 1987) ou replantées à proximité des maisons ${ }^{22}$.

\section{Espace de la cueillette éloignée}

18 Le cercle distal est celui des deesi fu mindii busi (« remèdes du cœur de la forêt »). Il s'agit d'un espace masculin, la grande forêt étant le domaine des hommes qui s'y rendent principalement pour chasser (illustration 5) et collecter des fruits de palmier comestibles. Ils ramènent des lianes sylvestres ou des écorces de grandes espèces ligneuses pour préparer leurs bita $a^{23}$, certaines écorces recherchées par les femmes pour leurs bains intimes (ketee), et des plantes pour le winti ${ }^{24}$ dont un bon nombre doivent justement leur efficacité magique au fait qu'elles proviennent de la "grande forêt " (van Andel et al., 2013). Certains cueilleurs de forêt se sont "professionnalisés", revendant des espèces récoltées en forêt afin de compléter les revenus de la chasse : les espèces récoltées sont vendues à des marchandes ou parfois en faisant du porte-àporte. Les gérants des bars, dans les villes, passent aussi parfois directement leurs commandes de plantes aux chasseurs pour la confection de leurs bita.

Illustration 5 - Chasseur ndjuka revenant de la chasse avec des plantes médicinales récoltées en forêt mature

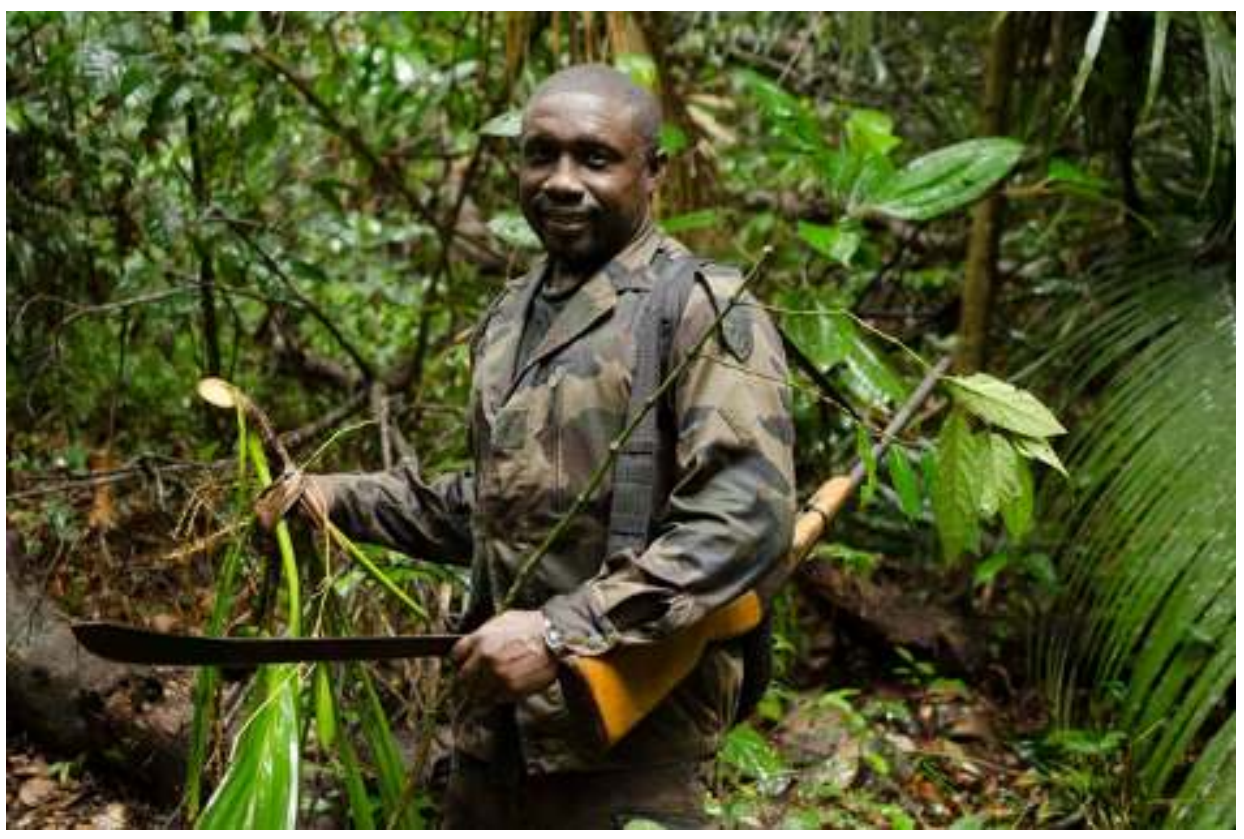

Auteur : M. Rapinski, novembre 2017. 


\section{L'adaptation des « cercles » de cueillette à l'univers urbain, l'exemple de Cayenne}

19 Dans la ville, les «cercles de cueillette " décrits chez les Ndjuka semblent encore opérants, mais avec des adaptations (illustration 6). Il est en effet possible d'établir une distinction entre l'espace domestique et l'espace intermédiaire en termes de distance avec le domicile: le premier est constitué de ses abords immédiats et du quartier d'habitation, là où l'on peut se rendre à pied de chez soi, et le second couvre des trajets plus longs nécessitant d'être véhiculé. Ainsi, alors que l'espace domestique de cueillette couvre toujours les zones autour de la maison, élargies au quartier, l'espace intermédiaire, lui, correspond plutôt à un archipel de lieux où l'on sait pouvoir trouver des plantes accessibles : herbacées rudérales ou plantes cultivées qui dépassent des jardins urbains. La frontière entre les deux espaces se fait en revanche plus floue si l'on considère les espèces collectées dans ces deux "cercles", puisque ce sont les mêmes. Les voies d'accès aux nouveaux quartiers périphériques et les bords des routes qui desservent Cayenne sont aussi exploités pour la collecte, faisant écho à l'espace réticulé des abattis et des bords de pistes en milieu rural, et composant, eux aussi, le cercle intermédiaire de cueillette qui correspond de facto à l'« espace vécu » des habitants (Frémont, 1999) ${ }^{25}$.

Mais les citadins vont également chercher des plantes médicinales dans les forêts et autres zones naturelles protégées périurbaines qui constituent, par leurs caractéristiques, le troisième cercle de cueillette. À Cayenne, les monts boisés qui parsèment le tissu urbain sont des lieux de collecte importants. Les populations «historiques » (Amérindiens, Businenge, Créoles) tirent profit de ces forêts car elles ont une bonne connaissance du milieu naturel local et des ressources qu'elles peuvent en tirer. Cependant, ces espaces sont également utilisés par les migrants, Haïtiens notamment, qui y cultivent de nombreux jardins). Ce dernier cercle est plus distant : les forêts urbaines forment des îlots épars dont la distribution est discontinue. Cette fragmentation de l'espace dans l'agglomération (illustration 6) donne lieu à des archipels d' "îles urbaines » (Saint-Laurent, 2000) dont la multipolarité est d'ailleurs également favorisée par l'allongement des déplacements urbains (Hubert and Delisle, 2010). 


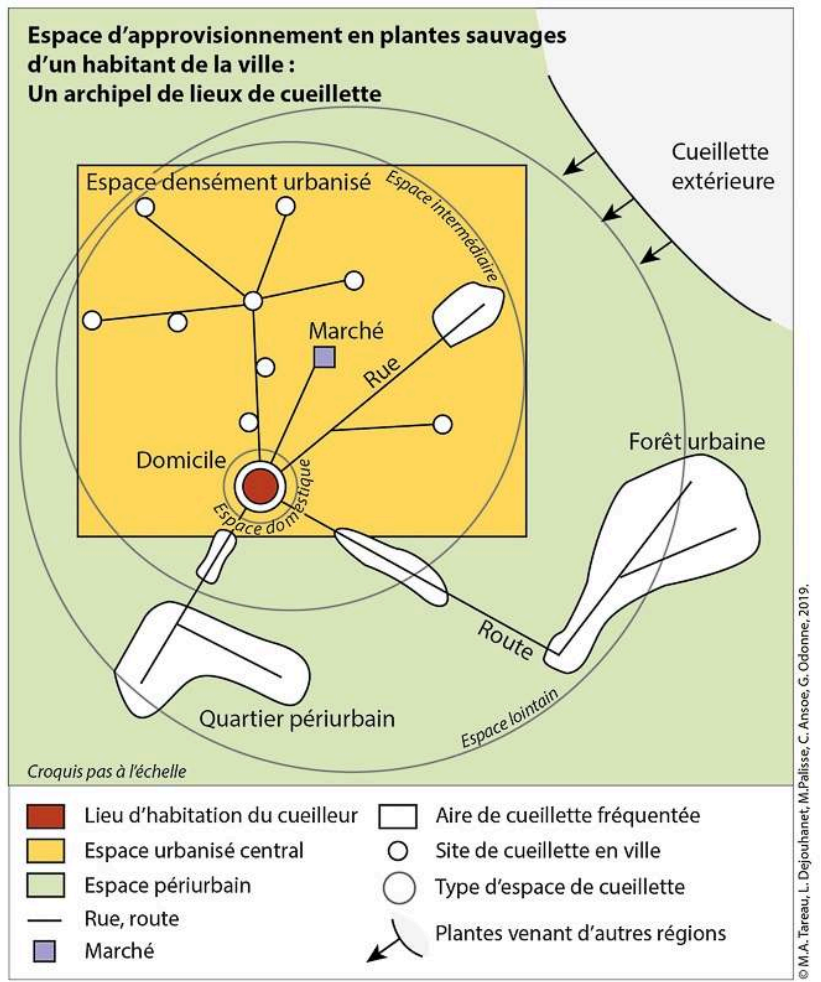

\section{Caractériser la cueillette de plantes médicinales.}

\section{L'hétérogénéité comme déterminant multifactoriel de la cueillette.}

Au-delà de cette organisation spatiale la diversité des situations de cueillette observées sur le terrain donne à penser que la notion d'« hétérogénéité » (Abreu et al., 2015; Ghimire et al., 2004 ; Ladio et Albuquerque, 2014) peut apporter une grille de lecture facilitant la compréhension de ces processus de collecte. L'hétérogénéité des pratiques de cueillette se trouve à la confluence de trois domaines d'hétérogénéité, sans doute complémentaires (illustration 7), qui agissent comme trois ingrédients contextuels déterminants :

- L'hétérogénéité culturelle se traduit par la présence de plusieurs groupes d'appartenance distincts au sein d'un même espace. Dans un espace multiculturel comme à Cayenne, chacun des groupes met en œuvre des choix de cueillette et d'espèces prélevées, associés à des usages qui lui sont propres. Ces pratiques ne sont cependant pas dénuées d'une certaine porosité entre les groupes.

- Les différences socio-économiques influencent également grandement la cueillette dans la mesure où les écarts de niveaux de vie induisent un accès différencié aux soins biomédicaux (Valmy et al., 2015) qui peuvent conduire à leur tour à des besoins de phytothérapie différents. De plus, les différences d'accès aux moyens de transports sont à l'origine d'une inéquitable répartition des ressources (Cerqueira, 2017). Enfin, le statut légal est également un facteur explicatif dans la mesure où les «sans-papiers » cueillent dans une aire moins étendue car ils se déplacent moins loin de leur domicile de peur de croiser la police. 
- Enfin, la diversité écologique est également un facteur à prendre en compte. La diversité des biotopes auxquels ont accès les habitants favorise l'émergence de types de collectes différents et accroît le nombre d'espèces pouvant potentiellement faire l'objet d'une cueillette. Des milieux variés et protégés sont donc propices à une pratique de cueillette plus diversifiée. A contrario, le milieu urbain (la ville doit en effet être considérée comme un système écologique à part entière, avec son climat, son hétérogénéité et sa propre biodiversité (Arnould et al., 2011) contraint les individus à des pratiques de l'espace urbain plus exploratoires et fragmentées, en cherchant des plantes dans les terrains vagues, les friches ou les petits espaces interstitiels.

Illustration 7 - Un système d'hétérogénéités pour expliquer les pratiques de cueillette

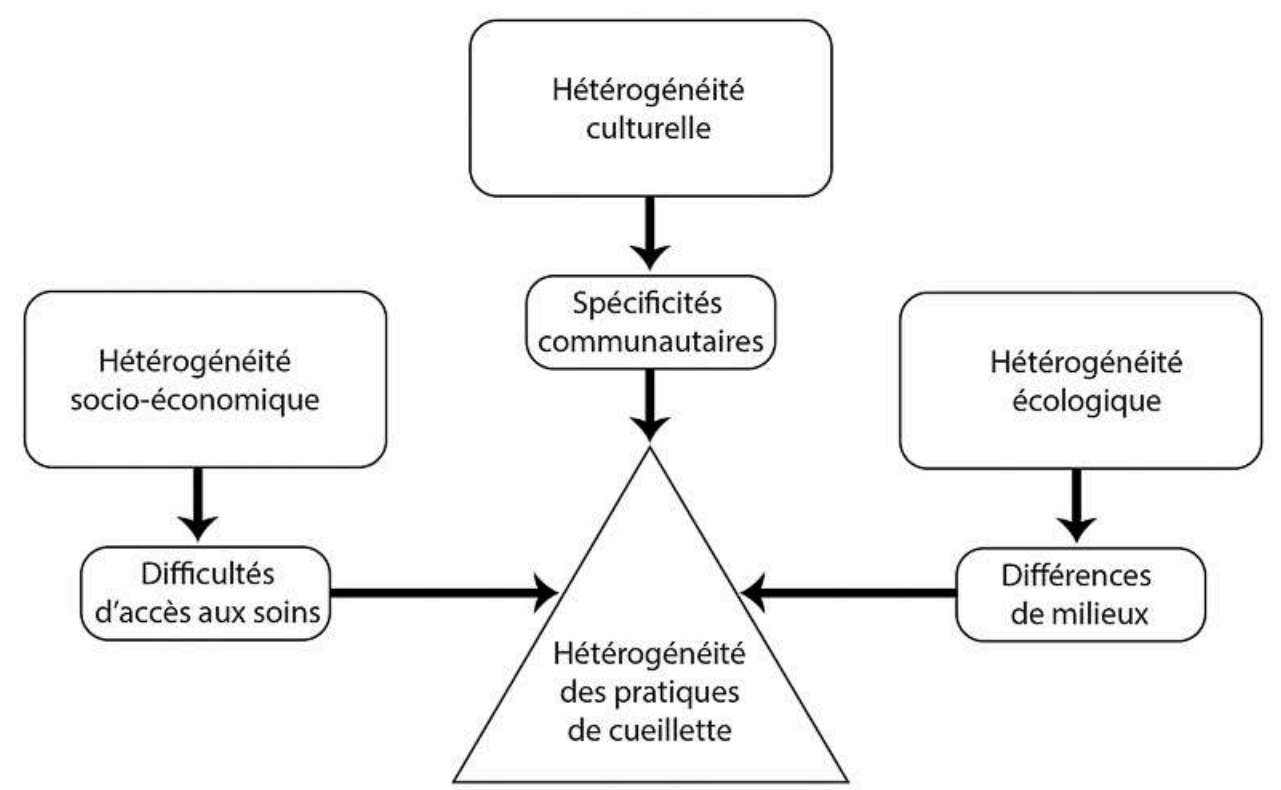

Ainsi, cette notion d'hétérogénéité permet de mieux appréhender les différences de cueillette qui peuvent être observées entre différents espaces. Si la diversité écologique peut sembler en apparence moins importante en milieu urbain, les conditions socioéconomiques et l'hétérogénéité culturelle des communautés de Cayenne composent une mosaïque de pratiques plus riches que dans la région du bas-Maroni. Si des populations businenge ont opéré une "relocalisation" en contexte urbain (Ladio et Albuquerque, 2014) de certaines de leurs pratiques culturelles de cueillette villageoise, c'est également le cas d'autres groupes (Haïtiens, Créoles...), ce qui donne naissance de facto à un enchevêtrement de pratiques de collectes qui se mêlent dans l'espace urbain. L'hétérogénéité des pratiques est ici principalement déterminée par la diversité des situations sociales et la diversité culturelle.

\section{Les objectifs de la cueillette : approche théorique}

Enfin, étudier les motivations qui animent le cueilleur semble aussi être une perspective pertinente afin d'élaborer un schéma explicatif de la cueillette de plantes médicinales (illustration 8). À un premier niveau d'explication, sont distinguées la collecte commerciale et la collecte domestique qui par essence n'ont pas les mêmes finalités. 
24 En ce qui concerne la collecte domestique, trois principaux objectifs se dégagent et sont associables aux trois domaines d'hétérogénéité précédemment définis. Tout d'abord, les personnes n'ayant pas un accès facile aux soins biomédicaux, pour des raisons économiques ("les médicaments de pharmacie coûtent chers»), administratives (migrants en situation irrégulière) ou géographiques (sites isolés) semblent cueillir davantage de plantes médicinales pour pallier leurs besoins de soins de santé primaire. Ceux qui cultivent déjà un certain nombre d'espèces à usage médicinal iront parfois collecter celles qui leur manquent pour préparer un remède domestique (ils peuvent aussi aller au marché), c'est ce que nous appelons la collecte d'appoint thérapeutique. Dans ce dernier cas, on observe une complémentarité entre les plantes cultivées dans le jardin et celles spontanées qui poussent en dehors. Enfin, certains besoins culturels spécifiques sont d'importants moteurs de collecte. Chez les Ndjuka, on l'a vu, la pratique du booko fu ketee ${ }^{26}$ est si courante qu'elle impulse une cueillette urbaine chez les femmes habitant en ville. Au sein de la communauté haïtienne, la préparation de bouteilles d'asosi encourage également les habitants à rechercher, protéger puis prélever les parties aériennes de Momordica charantia.

25 La cueillette commerciale est, elle, prioritairement motivée par un objectif de profit (Dejouhanet, 2014). La commercialisation des plantes médicinales ressort comme une spécificité du milieu urbain où elle répond à une demande. En milieu rural, l'abondance de la ressource rend majoritairement inutile cette monétisation des simples. En Guyane, peu d'unités de transformation des plantes médicinales existent et les filières de commercialisation à destination de ces petites entreprises sont très limitées et discrètes. Les marchands peuvent être amenées à transformer des produits pour en augmenter la valeur ajoutée.

Ces objectifs ${ }^{27}$ permettent de distinguer trois modalités de cueillette pouvant être pratiquées tant par les particuliers que par les marchands :

- Une collecte ciblée, fruit d'un déplacement intentionnel et dans un but défini. Le cueilleur se déplace pour chercher une plante dont il a besoin dans le but de préparer un remède précis ou pour répondre à une demande d'achat par une tierce personne. Cette collecte est par essence permise par le processus de cartographie mentale des plantes médicinales par les individus. À la campagne comme en ville, les espèces pouvant potentiellement faire l'objet d'un prélèvement sont quotidiennement repérées et leur emplacement est mémorisé par les habitant.

- Une collecte d'opportunité, non intentionnelle. Les plantes ramassées sont des plantes qui sont « croisées » par les personnes lors de leurs déplacements ou à l'occasion de moments particuliers (travail aux champs, partie de chasse...) et prélevées alors de façon opportuniste (et donc non anticipée).

- Une collecte complémentaire, qui est une collecte de pluriactivité ; elle est intentionnelle (on part avec l'intention de ramener des plantes) mais non définie (on ne sait pas encore quelles espèces on va trouver). Il s'agit typiquement de la cueillette qui a lieu lors des travaux à l'abattis ou lors de sorties de chasse ou de cueillette de fruits de palmiers. Cette collecte peut être domestique lorsque les plantes ramenées sont destinées aux proches du cueilleur ou commerciale lorsque celui-ci en profite pour obtenir un revenu supplémentaire. 


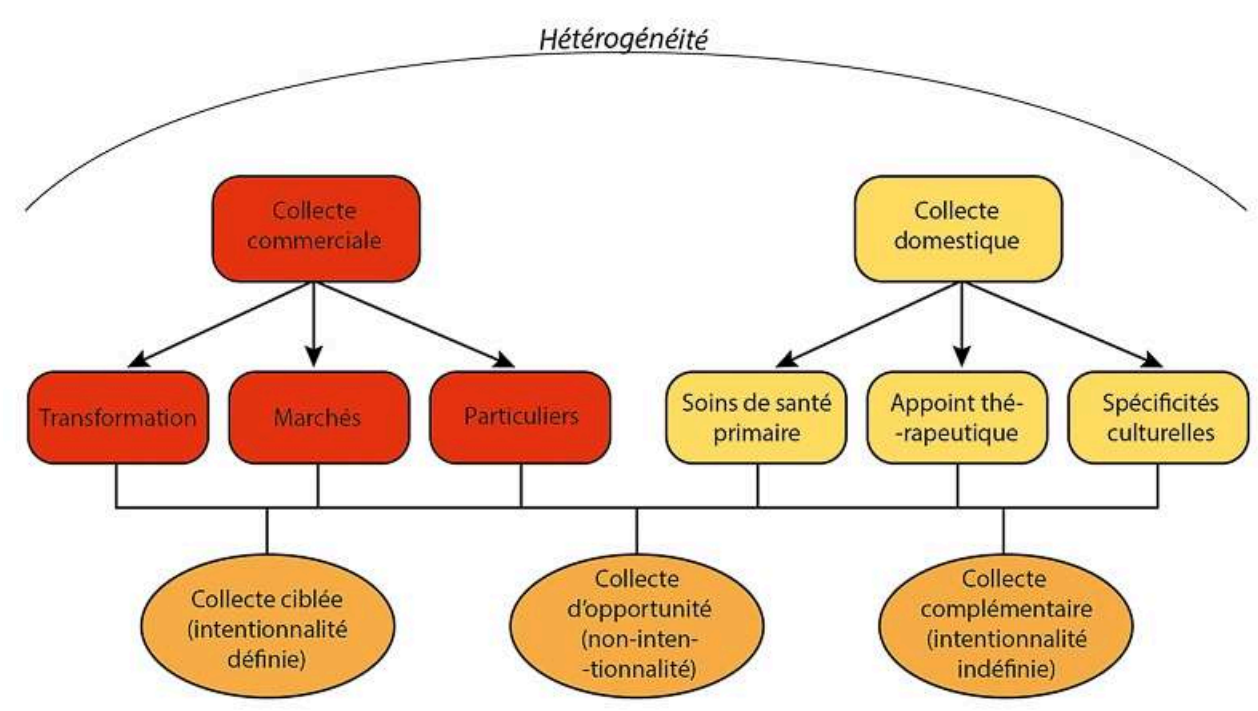

\section{Conclusion}

Le contexte multiculturel guyanais et l'évolution rapide de la population permettent une analyse très riche de socio-systèmes caractérisés par leur transformation. Les pratiques de cueillettes, souvent considérées comme des vestiges de niveaux de développement passés, montrent en Guyane leur vivacité : elles sont fréquentes, voyagent avec les populations et s'adaptent à de nouveaux environnements. Elles sont ici analysées au travers de plusieurs prismes conceptuels.

L'un d'entre eux, celui de l'hétérogénéité, permet de comprendre la multiplicité des pratiques. Les caractéristiques socio-culturelles des populations et écologiques du milieu induisent une pluralité des pratiques de collecte, dépendante également du contexte économique dans lequel ces activités ont lieu: les pratiques sont le fruit de combinaisons particulières entre les trois domaines d'hétérogénéité ainsi définis.

La compréhension spatiale des pratiques est le deuxième niveau d'analyse : trois zones, proximale/domestique, intermédiaire et distale/périphérique ont pu être définies en fonction à la fois de la proximité par rapport au foyer, des habitudes de parcours et des représentations spirituelles associées. Les plantes qui sont cueillies dans ces trois zones diffèrent autant du point de vue botanique qu'en termes d'indications thérapeutiques, puisque leurs propriétés sont intimement liées à ces représentations culturelles. Ces «cercles » théoriques, centrés sur le foyer du cueilleur, persistent de façon partielle lors de la transition entre milieu villageois et milieu urbain, avec des ajustements. Ainsi l'équilibre entre les trois niveaux semble-t-il se déplacer au profit d'un très vaste espace réticulaire de la zone intermédiaire, à géométrie variable en fonction des moyens de déplacements des acteurs.

Enfin, les objectifs qui sous-tendent les différentes collectes offrent une autre vision de ces pratiques. Les cueillettes peuvent se diviser entre cueillette ciblée, cueillette d'opportunité et cueillette complémentaire selon que l'action est planifiée ou non et que l'objet de la cueillette est défini ou non. La dichotomie entre cueillette commerciale 
et cueillette domestique reste encore peu pertinente en Guyane, mais les changements de mode de vie et les déplacements de population pourraient accroître les besoins en plantes de populations qui ne peuvent plus les cueillir elles-mêmes, entraînant le développement de nouvelles filières commerciales, comme c'est le cas aujourd'hui au Suriname voisin.

31 Si la nature dans la ville est devenue un enjeu de recherche important en sciences humaines et sociales, les perspectives en termes de cueillette de plantes médicinales invitent à prendre davantage en considération dans l'espace public ces usages différenciés du milieu végétal sauvage. Le rapport à la nature urbaine, la place du végétal en ville- trop souvent cantonné à des espaces verts aménagés-, et la volonté de rationaliser sa présence dans le paysage sont à repenser. Entre fonctionnalité pour des populations défavorisées pour qui cette collecte permet de répondre en partie aux besoins de santé primaire et nouvelles aspirations écologiques des citadins dans leurs modes d'habiter la ville, ce travail alimente les réflexions sur la variété des usages de l'espace urbain, sur les représentations contemporaines de la ville et des ressources qu'elle abrite et sur les enjeux associés à une gestion différenciée de la nature en ville.

\section{BIBLIOGRAPHIE}

Abreu D. B. de O., Santoro F. R., Albuquerque U. P. de, Ladio A. H., Medeiros P. M. de, 2015. Medicinal plant knowledge in a context of cultural pluralism: A case study in Northeastern Brazil. Journal of Ethnopharmacology, vol. 175, n 1, p. 124-130.

Albert B., Le Tourneau F.-M., 2007. Ethnogeography and resource use among the Yanomami: Toward a model of "reticular space." Current Anthropology, vol. 48, n 4, p. 584-592.

Arnould P., Cieslak C., 2004. Presentation of natural objects in Paris and Warsaw: remarkable trees of two periurban woods. Natures Sciences Sociétés, vol. 12, n² 2, p. 157-171.

Arnould P., Le Lay Y.-F., Dodane C., Méliani I., 2011. La nature en ville : l'improbable biodiversité. Géographie Économie Société, vol. 13, n 1, p. 45-68.

Auburtin R., 2007. La production du logement en Guyane : contraintes réglementaires et contrepratiques populaires. In Man Lam Fouck S. (ed.), Comprendre la Guyane d'aujourd'hui. Un département français dans la région des Guyanes. Matoury, Ibis Rouge.

Auburtin R., 2006. De Cayenne à Kourou, singularité des villes françaises de Guyane : entre habitat d'ordonnance et habitat auto-construit, la question du logement. Thèse de Doctorat, Paris 3, $484 \mathrm{p}$.

Bahuchet S., 1997. Un style de vie en voie de mutation. Considérations sur les peuples des forêts denses humides. Civilisations. Revue internationale d'anthropologie et de sciences humaines, vol. 44, $\mathrm{n}^{\circ} 1$, p. 16-31.

Baudry S., 2015. Gestion environnementale de la voie publique par les citoyens : mise en relation des discours officiels et des pratiques quotidiennes locales à Paris et New York City. In Beringuier P., Frédérique Blot F., Desailly B., Saqalli M. (dir.), Environnement, politiques publiques et pratiques locales. Paris, L'Harmattan, 2015, p. 50-68. 
Baudry S., Scapino J., Rémy E., 2014. L'espace public à l'épreuve des jardins collectifs à New York et Paris. Géocarrefour: Revue de géographie de Lyon, vol. 89, n 1, p. 41-51.

Bourdeau-Lepage L., Vidal R., 2012. Nature urbaine en débat : à quelle demande sociale répond la nature en ville? Demeter Journal, vol. 13, $n^{\circ} 1$, p. 195-210.

Bourgarel S., 1990. Les réfugiés surinamiens en Guyane. Etudes Créoles, vol. 13, n 2, p. 7-29.

Burgess E.W., 1929. Urban areas. Chicago: An experiment in social science research, vol. $12, \mathrm{n}^{\circ} 1$, p. 113-138.

Bussmann R.W., Sharon D., 2009. Markets, Healers, Vendors, Collectors: The Sustainability of Medicinal Plant Use in Northern Peru. Mountain Research and Development, vol. 29, n 1, p. 128-134.

Cabrera L., 2003. La Forêt et les Dieux. Religions afro-cubaines et médecine sacrée à Cuba. Paris, Jean Michel Place, $603 \mathrm{p}$.

Carde E., 2006. Les discriminations selon l'origine dans l'accès aux soins. Etude en France métropolitaine et en Guyane française. Thèse de Doctorat, Université Paris Sud - Paris XI, 541 p.

Carney J., Rosomoff R.N., 2011. In the shadow of slavery: Africa's botanical legacy in the Atlantic world. USA, University of California Press, 296 p.

Carney J.A., 2003. African traditional plant knowledge in the circum-Caribbean region. Journal of Ethnobiology, vol. 23, n², p. 167-186.

Cerqueira E.V., 2017. Les inégalités d'accès aux ressources urbaines dans les franges périphériques de Belo Horizonte (Brésil) : quelles évolutions ? EchoGéo [En ligne], n 39. http:// journals.openedition.org/echogeo/14856

Ceuterick M., Vandebroek I., Torry B., Pieroni A., 2008. Cross-cultural adaptation in urban ethnobotany: The Colombian folk pharmacopoeia in London. Journal of Ethnopharmacoly, vol. 120, $\mathrm{n}^{\circ}$ 3, p. 342-359.

Clastres P., 1972. Chronique des Indiens Guayaki. Ce que savent les Aché, chasseurs-nomades du Paraguay. Paris, Plon, 320 p.

Clément G., 2014 [2004 : $1^{\text {ère }}$ ed.]. Manifeste du Tiers paysage. Paris, Sens \& Tonka, 48 p.

Collomb G., Jolivet M.-J., 2008. Histoires, identités et logiques ethniques : Amérindiens, Créoles et NoirsMarrons en Guyane. Paris, Éditions du CTHS, 221 p.

Dejouhanet L., 2014. Secteur informel et réseaux de commercialisation des plantes médicinales au Kerala (Inde). Économie Rurale [En ligne], n 343, p. 53-70. https://journals.openedition.org/ economierurale/4447

Descola P., 2004a. Le sauvage et le domestique. Communications, vol. 76, $\mathrm{n}^{\circ}$ 1, p. 17-39.

Descola P., 2004b. La Nature domestique : Symbolisme et praxis dans l'écologie des Achuar. Paris, Les Editions de la MSH, $234 \mathrm{p}$.

Descola P., 2001. Par-delà la nature et la culture. Le Débat, vol. 114, nº 3, p. 86-101.

Descola P., 1993. Les Affinités sélectives : Alliance, guerre et prédation dans l'ensemble jivaro. L'Homme, vol. 33, n 12, p. 171-190.

Dupuy F., 2007. Dynamiques interethniques dans le haut Maroni. In Léglise I., Migge B., (ed.), Pratiques et Représentations Linguistiques en Guyane. Regards Croisés. Paris, IRD Éditions, p. 107-117.

Ellena R., Quave C.L., Pieroni A., 2012. Comparative Medical Ethnobotany of the Senegalese Community Living in Turin (Northwestern Italy) and in Adeane (Southern Senegal). Evidence- 
Based Complementary and Alternative Medicine [En Ligne], vol. 2012. http://dx.doi.org/ $10.1155 / 2012 / 604363$

Fleury M., 2015. Plantes médicinales chez les Aluku (Boni) du Haut-Maroni : origine, symbolique

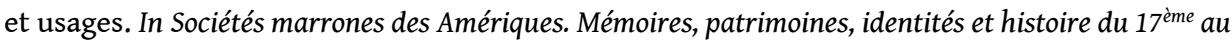
$20^{\text {ème }}$ siècles. Matoury, Ibis Rouge.

Fleury M., 1996. Végétaux utilisés pour l'hygiène intime des femmes Aluku en Guyane française : interprétation culturelle et intérêt pharmacologique. In Schröder E., Balansard G., Cabalion P., Fleurentin J., Mazars G., Ethnopharmacologie. Sources, méthodes, objectifs. Paris, Société française d'Ethnopharmacologie - Editions de l'ORSTOM, p. 178-186.

Fleury M., 1991. Busi-Nenge, les hommes-forêt : Essai d'ethnobotanique chez les Aluku (Boni) en Guyane française. Thèse de doctorat, Université Paris 6, $392 \mathrm{p}$.

Frémont A., 1999. La région, espace vécu. Paris, Flammarion, 288 p.

Gardel A., 2001. Les paysages urbains de l'île de Cayenne, Guyane Française. Mappemonde, vol. 63, $\mathrm{n}^{\circ} 3$, p. 16-21.

Garine-Wichatitsky É., 1997. Sauvage ou domestique ? Remarques sur l'inventaire des plantes à brèdes chez les Gimbe et les Duupa du Nord-Cameroun In Barreteau D., Dognin R., Von Graffenried C., Homme Milieu Végétal Dans Bassin Lac Tchad, Paris, ORSTOM Editions, p. 311-326.

Ghimire S.K., Doyle M., Yildiz A., 2004. Heterogeneity in ethnoecological knowledge and management of medicinal plants in the Himalayas of Nepal: implications for conservation . Ecology and Society, [En Ligne], Volume 9, $n^{\circ} 3$. http://www.ecologyandsociety.org/vol9/iss3/art6/ Godinho I., 1984. Les définitions d'« adventice » et de «mauvaise herbe », Weed Research, vol. 2, $\mathrm{n}^{\circ} 24$, p. $121-125$.

Gorgeon C., 1985. Immigration clandestine et bidonvilles en Guyane, les Haïtiens à Cayenne. Revue européenne des migrations internationales. vol. 1, $\mathrm{n}^{\circ} 1$, p. 143-158.

Grafmeyer Y., Joseph I., 1984, L'École de Chicago. Naissance de l'écologie urbaine. Paris, Aubier, 378 p. Grimé W.E., 1979, Ethno-botany of the Black Americans. USA, References Publications, 237 p. Harlan J.R., et Technique A. de C.C., 1987. Les plantes cultivées et l'homme. Paris, Payot, 414 p. Haudricourt A.-G., Hédin L., 1987. L’Homme et les plantes cultivées. Paris, Gallimard, 290 p.

Havinga R.M., 2006. Harvest of Medicinal Plants in Surinamese Maroon Society: Implications for sustainability. Mémoire de Maîtrise, Université d'Utrecht, 59 p.

Hubert J.-P., Delisle F., 2010. L'allongement des déplacements quotidiens contribue à l'émergence d'espaces urbains multipolaires, tandis que la mobilité baisse au centre des grandes agglomérations. La Revue du CGDD, vol. 3, n² 2, p. 49-64.

Hurpeau B., 2012. Panorama de la population immigrée en Guyane. France, INSEE Antilles-Guyane, $21 \mathrm{p}$.

INSEE, 2014. Recensement de la population en Guyane - 252338 habitants au 1er janvier 2014 [En Ligne]. https://www.insee.fr/fr/statistiques/2540209

Jolivet M.-J., 1982. La question créole. Essai de sociologie sur la Guyane française. Paris, Editions de l'ORSTOM, $495 \mathrm{p}$.

Ladio A.H., Albuquerque U.P., 2014. The concept of hybridization and its contribution to urban ethnobiology. Ethnobiology and Conservation, vol. 3, p. 1-9. 
Landy F., Belaidi N., Sada K.-H.G., 2017. Les espaces protégés urbains, vecteurs de justice ou d'injustice pour les populations autochtones? Les cas de Xochimilco et des parcs nationaux de Mumbai et du Cap. Justice spatiale/ Spatial Justice [En ligne], n 11. http://www.jssj.org/article/lesespaces-proteges-urbains-vecteurs-de-justice-ou-dinjustice-pour-les-populations-autochtonesles-cas-de-xochimilco-et-des-parcs-nationaux-de-mumbai-et-du-cap/

Lenaerts M., 2006. Ontologie animique, ethnosciences et universalisme cognitif. Le regard ashéninka. L'Homme. Revue française d'anthropologie, vol. 179, n 33, p. 113-139.

Léobal C., 2013. Saint-Laurent du Maroni : une porte sur le fleuve. Matoury, Ibis Rouge éditions, 216 p.

Marc J.-V., 2007. Le Végétal dans les espaces urbains et périurbains des petites antilles : Le cas de Fort-De-France. Thèse de Doctorat, Université des Antilles et de la Guyane, 337 p.

Moomou J., 2002. Le monde des Marrons du Maroni en Guyane (1772-1860). La naissance d'un peuple : les Boni. Matoury, Ibis Rouge Editions, 222 p.

Nguenang G.M., Fedoung E.F., Nkongmeneck B.A., 2010. Importance des forêts secondaires pour la collecte des plantes utiles chez les Badjoué de l'Est Cameroun. Tropicultura, vol. 28, $\mathrm{n}^{\circ} 4$, p. 238-245.

Odonne G., Bourdy G., Beauchêne J., Houël E., Stien D., Chevolot L., Deharo E., 2007. From Toniccups to Bitter-cups: Kwasi bita beker from Suriname: Determination, past and present use of an ancient galenic artefact. Journal of Ethnopharmacology, vol. 110, n² 2, p. 318-322.

Ososki A.L., Balick M.J., Daly, D.C., 2007. Medicinal plants and cultural variation across Dominican rural, urban, and transnational landscapes. In Pieroni A., Vandebroek I., Traveling Cultures and Plants: The Ethnobiology and Ethnopharmacy of Migrations. USA, Berghahn Books.

Palisse M., 2016. Les pratiques agricoles des migrants haïtiens en Guyane : entre insertion et stigmatisation. In Mobilités, ethnicités, diversité culturelle : La Guyane entre Suriname et Brésil. Matoury, Ibis rouge Editions.

Palisse M., 2013. Libres de savane. Pratiques et imaginaires autour des savanes de Guyane. Rapport de recherche, Université des Antilles-Guyane, $62 \mathrm{p}$.

Park R.E., Burgess, E.W., McKenzie, R.D., 1984. The city. USA, University of Chicago Press, 250 p.

Pellegrini P., Baudry S., 2014. Streets as new places to bring together both humans and plants: examples from Paris and Montpellier (France). Social \& Cultural Geography, Volume 15, $\mathrm{n}^{\circ} 8$, p. 871-900.

Piantoni F., 2011. Migrants en Guyane. Arles, Actes Sud. Editions, 176 p.

Price R., 2013a. Les Premiers Temps : la conception de l'histoire des Marrons Saamaka. Paris, Vents d'ailleurs, $304 \mathrm{p}$.

Price R., 2013b. « The Maroon Population Explosion: Suriname and Guyane ». New West Indian Guide/Nieuwe West-Indische Gids. Volume 87, n 3, p. 323-327.

Price R., Mintz S.W., 2013. The birth of African-American culture. USA, Beacon Press, 144 p.

Price R., 2010. Voyage avec Tooy. Histoire, mémoire, imaginaire des Amériques Noires. Paris, Vents d'ailleurs, $520 \mathrm{p}$.

Price R., Price S., 2008, Les Marrons. Paris, Vents d'ailleurs, 128 p.

Romanovski Z., Piantoni F., 2009. Les stratégies d'accès au logement des Haïtiens dans l'agglomération de Cayenne comme facteurs de restructuration urbaine. L'Espace Politique. Revue 
en ligne de géographie politique et de géopolitique [En Ligne], $\mathrm{n}^{\circ} 3 \mathrm{http} / /$ journals.openedition.org/ espacepolitique/1009; DOI : 10.4000/espacepolitique.1009

Roulleau-Berger L., Lu S., 2003. Les provinciaux à Shanghaï : Formes d'inscriptions urbaines et économiques des migrants dans la ville. Les Annales de la Recherche Urbaine, vol. 93, n 1, p. 48-56.

Saint-Laurent D., 2000. Approches biogéographiques de la nature en ville : parcs, espaces verts et friches. Cahiers de géographie du Québec, vol. 122-44, p. 147-166.

Sauvain M., Vernon D., Fleury M., Jamet P., Daniel R., 1988. Système de santé moderne et pratiques traditionnelles de santé chez les Noirs Marrons de Guyane et du Surinam : rapport d'activité. Paris, ORSTOM Editions, 83 p.

Sukopp H., Hejny S., 1990. Plants and plant communities in urban environments. The Netherlands, SPB Academic Publishing, $282 \mathrm{p}$.

Tareau M.A., Palisse M., Odonne G., 2017. As vivid as a weed... Medicinal and cosmetic plant uses amongst the urban youth in French Guiana. Journal of Ethnopharmacology, vol. 203, n 1, p. 200-213.

Tareau M.A., Odonne G., Fozzani J., Palisse M., à paraître. Pratiques de cueillette urbaine des plantes médicinales dans l'île de Cayenne. In Roger D., Guibert J-S., Habiter la ville ou la fabrique de la cité (vol 2). Rennes, Presses Universitaires de Rennes.

Thomas W.I., Znanieck, F., 1918. The Polish peasant in Europe and America: Monograph of an immigrant group. USA, University of Chicago Press, $317 \mathrm{p}$.

Valadeau C., 2016. Agir en secret : un regard sur la circulation des usages associés aux souchets des jardins, aux fougères des sous-bois et aux caladions des berges chez les Yanesha (Haute Amazonie péruvienne). Bulletin de l'Institut français d'études andines, vol. 45, n 1, p. 173-192.

Valmy L., Gontier, B., Parriault, M.C., Van Melle, A., Pavlovsky, T., Basurko, C., Grenier, C., Douine, M., Adenis, A., Nacher, M., 2015. Prevalence and predictive factors for renouncing medical care in poor populations of Cayenne, French Guiana. BMC health services research, vol. 16, $\mathrm{n}^{\circ} 1$, p. 34-45.

van Andel T., 2016. The Reinvention of Household Medicine by Enslaved Africans in Suriname. Social History of Medicine, vol. 29, $\mathrm{n}^{\circ}$ 4, p. 676-694.

van Andel T., Behari-Ramdas J., Havinga R., Groenendijk S., 2007. The medicinal plant trade in Suriname. Ethnobotany Research and Applications, vol. 5, nº 1, p. 351-372.

van Andel T., Carvalheiro L.G., 2013. Why Urban Citizens in Developing Countries Use Traditional Medicines: The Case of Suriname. Evidence-Based Complementary and Alternative Medicine [En ligne], n 2013 . http://dx.doi.org/10.1155/2013/687197

van Andel T., de Korte S., Koopmans D., Behari-Ramdas J., Ruysschaert S., 2008. Dry sex in Suriname. Journal of Ethnopharmacology, vol. 116, n 1, p. 84-88.

van Andel T., Mitchell S., Volpato G., Vandebroek I., 2012. In search of the perfect aphrodisiac: parallel use of bitter tonics in West Africa and the Caribbean. Journal of Ethnopharmacology, vol. $143, n^{\circ} 3$, p. 840-850.

van Andel T., Ruysschaert S., Putte K.V. de, Groenendijk S., 2013. « What makes a plant magical? Symbolism and sacred herbs in Afro-Surinamese winti rituals » in African ethnobotany in the Americas. USA, Springer, 247-284.

van Andel T., Westers P., 2010. Why Surinamese migrants in the Netherlands continue to use medicinal herbs from their home country. Journal of Ethnopharmacoly, vol. 127, n 3, p. 694-701. 
van Andel T.R., van't Klooster C.I., Quiroz D., Towns A.M., Ruysschaert S., van den Berg M., 2014. Local plant names reveal that enslaved Africans recognized substantial parts of the New World flora. Proceedings of the National Academy of Sciences, vol. 111-50, p. 346-353.

van Velzen H.U.E.T., van Wetering W., 1988. The Great Father and the Danger: religious cults, material forces, and collective fantasies in the world of the Surinamese Maroons. The Netherlands, Foris Publications, $88 \mathrm{p}$.

van't Klooster C.I., Haabo V., Ruysschaert S., Vossen T., van Andel T.R., 2018. Herbal bathing: an analysis of variation in plant use among Saramaccan and Aucan Maroons in Suriname. Journal of Ethnobioly Ethnomedicine, vol. 14, $\mathrm{n}^{\circ} 1$, p. 20-33.

Vernon D., 1993. Choses de la forêt. Identité Thérapie Chez Noirs Marrons Ndjuka Surinam ; In Jolivet M-J, Rey-Hulman D., Jeux d'identités. Études comparées à partir de la Caraibe. Paris, L'Harmattan, p. 261-281.

Vernon D., 1992. Les représentations du corps chez les Noirs Marrons Ndjuka du Surinam et de la Guyane française. Paris, Editions de l'ORSTOM, 95 p.

Voeks R.A., 2007. Are women reservoirs of knowledge? Gender, ethnobotany and globalization in northeast Brazil. Singapore Journal of Tropical Geography, vol. 28, n 1, p. 7-20.

Volpato G., Godinez D., Beyra A., 2008. Migration and ethnobotanical practices: the case of tifey among haitians immigrants in Cuba. Human Ecology, vol. 37, n 1, p. 43-53.

Vossen T., Towns A., Ruysschaert S., Quiroz D., van Andel T., 2014. Consequences of the transAtlantic slave trade on medicinal plant selection: plant use for cultural bound syndromes affecting children in Suriname and Western Africa. PloS One [En ligne], $n^{\circ} 11$. https://doi.org/ 10.1371/journal.pone.0112345

Wehi Priscilla M., Wehi William L., 2010. Traditional Plant Harvesting in Contemporary Fragmented and Urban Landscapes. Conservation Biology, vol. 24, nº 2, p. 594-604.

Wiel M., 1999. La transition urbaine : villes bonnes à vivre, villes invivables. Revue semestrielle MAUSS, vol. 39, $\mathrm{n}^{\circ} 14$, p. 175-184.

Wooding C.J., 1979. Traditional Healing and Medicine in Winti: A Sociological Interpretation. African Issues, vol. $9, \mathrm{n}^{\circ}$ 3, p. 35-40.

Zeder M.A., 2006. Central questions in the domestication of plants and animals. Evolutionary Anthropology. Issues, News, and Reviews, vol. 15, n 3, p. 105-117.

\section{NOTES}

1. En Occident, selon Ph. Descola, le paysage romain et les valeurs qui lui sont associées avaient dessiné la frontière d'une polarisation entre le sauvage et le domestique dont nous sommes encore tributaires aujourd'hui (Descola, 2004a).

2. Jusqu'au $1^{\mathrm{er}}$ juillet 2017 , entrée en vigueur des décrets d'application de la loi réglementant la recherche sur les connaissances traditionnelles associées aux ressources génétiques. Les données collectées ultérieurement ne s'intéressaient qu'à la désignation des espèces collectées, sans y associer ni indications thérapeutiques ni localisation précise.

3. Un certain nombre de collines boisées parsèment l'espace urbain de l'agglomération de Cayenne.

4. Il s'agit du terme guyanais pour désigner les marais et savanes inondables.

5. C'est-à-dire née étrangère à l'étranger et résidant en France (Hurpeau, 2012). 
6. De son nom vernaculaire haïtien (également yesken d'ailleurs). Les Créoles guyanais l'appellent plutôt sorosi, les businenge busi sopolopo et les Créoles antillais paroka ou manjé kouli.

7. En Guyane, un amer est un alcool médicinal composé d'une mixture de plantes - souvent amères - mises à macérer dans du rhum.

8. Transmis notamment sur le mode amazonien des «amitiés cérémonielles » décrites par Ph. Descola (1993).

9. On parle de booko fu ketee, «cueillir pour le ketee » (de l'anglais kettle, bouilloire, utilisée par les femmes businenge pour faire bouillir les plantes destinées aux bains).

10. Il s'agit sans doute de l'une des religions afro-américaines les moins connues (van Andel et al., 2013), mêlant à des croyances d'origines africaine et européenne de nombreux traits amérindiens (objets cérémoniels, divinités, flore rituelle...).

11. Quelques exemples de pré-domestication récents ont tout de même pu être constatés, notamment en ce qui concerne deux espèces: Begonia glabra Aubl. (koo ati) et Renealmia alpinia (Rottb.) Maas (masusa).

12. Notons que chez les Ndjuka, certaines plantes de la forêt sont considérées sacrées - et, in extenso, magiques - parce qu'elles ont contribué à la survie de leurs ancêtres fugitifs (van Andel et al., 2013).

13. D'ailleurs, l'utilisation de l'ordre social propre au groupe pour penser les rapports des nonhumains entre eux, telle que le suggère $\mathrm{Ph}$. Descola (2001), se retrouve ici puisque certaines plantes particulièrement importantes sont considérées comme les kapiten des plantes (Fleury, 2015), en référence aux kapiten (chefs) qui dirigent les konde ("grands villages ») businenge (Collomb et Jolivet, 2008).

14. Chez qui Descola décrit une segmentation de l'espace en secteurs de socialisation décroissante.

15. M. Lenaerts distingue lui aussi trois cercles concentriques : « au plus loin » où se trouvent les plantes les plus «fortes » utilisées par les chamanes, « au plus près » où se trouvent les espèces cultivées et « la zone intermédiaire » où l'on trouve les espèces de jachère et de forêt moyenne qui sont les plus utilitaires.

16. Pour reprendre la formule de P. Clastres (1972) chez les Marrons également « il y a un espace masculin et un espace féminin, respectivement définis par la forêt où les hommes chassent et par le campement où règnent les femmes ".

17. De plus, les Amérindiens ont l'habitude de cultiver autour de leurs habitations un certain nombre d'espèces propitiatoires visant à refouler les mauvais esprits (Valadeau, 2016).

18. Plantes qui croissent dans un milieu modifié par l'homme.

19. Il s'agit, chez les Ndjuka, d'un interdit formel : les femmes menstruées ne doivent pas toucher les remèdes car ils ne fonctionneraient alors pas (Vernon, 1992).

20. Très utilisé également par les Créoles ruraux qui le nomment yanman.

21. Les adventices sont des plantes sauvages qui poussent au milieu des cultures (Godinho, 1984).

22. Ce dernier exemple correspond typiquement, selon la classification de Zeder (2006), à la catégorie des espèces végétales domestiquées. Selon l'auteur cité, il s'agit des plantes indigènes dont la culture autour des habitations fait l'objet de tentatives plus ou moins fructueuses.

23. De l'anglais bitter, amer. Comme vu précédemment, il s'agit d'alcoolatures médicinales de plantes amères consommées par les hommes ndjuka.

24. Winti désigne l'ensemble des croyances religieuses afro-surinamaises.

25. L'un de nos informateurs, véhiculé, nous expliquait que ses points de collecte correspondent aux différents quartiers qu'il a l'habitude de fréquenter et qui ne sont pas forcément connectés entre eux d'un point de vue spatial : il cueille indifféremment dans les quartiers où il se rend pour travailler, aller voir ses proches, jouer au football ou emmener ses enfants à l'école...

26. Littéralement : « cueillir pour le bain intime ». 
27. Dans d'autres contextes, en particulier les villes du nord, on pourrait sans doute compléter ces objectifs de cueillette de plantes médicinales par d'autres finalités, comme la collecte récréative ou la collecte à visée pédagogique.

\section{RÉSUMÉS}

La cueillette des plantes médicinales en Guyane française est une pratique relativement méconnue, sous-estimée et souvent perçue comme anecdotique ou insignifiante. Pourtant, tous les jours, des personnes peuvent être vues ramassant des plantes sur les bords de route, pour leur propre usage ou, dans de moindres proportions, pour les vendre. Cet article vise à caractériser ces pratiques de cueillette des plantes médicinales et leur diversité et à souligner la pertinence de l'entrée par ces plantes pour interroger le fonctionnement de sociétés sujettes à des transformations rapides et désignées ici comme « en transition ». À la lumière de deux études de cas, une sur la cueillette urbaine dans l'agglomération de Cayenne et l'autre en zone rurale le long du bas-Maroni, cet article permet de repenser les relations nature-société-ville. En observant les spécificités socio-culturelles influençant les modalités de cueillette, il propose un schéma conceptuel de ces pratiques en mobilisant les idées d'hétérogénéité et de finalité comme concepts explicatifs.

The gathering of medicinal plants in French Guiana is a relatively unknown practice, underestimated and often perceived as an anecdotic or insignificant one. However, everyday people can be seen harvesting plants on the road sides, for their own needs or in small proportions, for selling. This paper aims to characterise these medicinal plants' collection practices, their diversity and their relevance for questioning the functioning of societies, which are subjected to rapid transformations and are therefore designed here as "transitional". Through two case studies, one on urban collection in Cayenne city and one in the rural area along the low Maroni river, this paper analyses the connection between societies, nature and urban dynamics. By observing the socio-cultural specificities influencing collection modes, we propose a conceptualization scheme of collection practices, through the explanatory concepts of heterogeneity and purpose.

\section{INDEX}

Mots-clés : cueillette, plante médicinale, Guyane française, Businenge

Keywords : gathering, medicinal plant, French Guiana, Businenge

Thèmes : Sur le Champ - Sur le Terrain

\section{AUTEURS}

\section{MARC-ALEXANDRE TAREAU}

Marc-Alexandre Tareau, tareau.marc.alexandre@gmail.com, est doctorant en ethnobotanique et en anthropologie de la santé à l'Université de Guyane. Il a récemment publié :

- Tareau, M.-A., Palisse, M., Odonne, G., 2017. As vivid as a weed... Medicinal and cosmetic plant 
uses amongst the urban youth in French Guiana. Journal of Ethnopharmacology, $n^{\circ} 203$, p. 200-213. - Tareau M.A., Odonne G., Fozzani J., Palisse M., 2019. Pratiques de cueillette urbaine des plantes médicinales dans l'île de Cayenne. Rennes, Presses Universitaires de Rennes, sous presse.

\section{LUCIE DEJOUHANET}

Lucie Dejouhanet, lucie.dejouhanet@orange.fr, est maître de conférences en géographie, Université des Antilles, AIHP-GEODE EA 929. Elle a récemment publié :

- Dejouhanet L., 2014. Supply of Medicinal Raw Materials. The Achilles Heel of Today's Manufacturing Sector for Ayurvedic Drugs in Kerala. Asian Medicine, vol. 7, n 3, p. 206-235.

- Dejouhanet L., 2014. Secteur informel et réseaux de commercialisation des plantes médicinales au Kérala (Inde). Économie rurale, $\mathrm{n}^{\circ} 343$, p. 53-70.

- Dejouhanet L., 2017. Tourism in the mountains of Central Kerala (South India): at the Crossroads of attitudes toward forest populations. Journal of Alpine Research, vol. 105, $\mathrm{n}^{\circ} 3$ / Le tourisme dans les montagnes du centre du Kérala (Inde du Sud) : à la croisée des regards posés sur les populations forestières. Journal de Géographie Alpine, vol. 105, nº 3.

\section{GUILLAUME ODONNE}

Guillaume Odonne, guillaume.odonne@cnrs.fr, est chargé de recherche au CNRS, UMR LEEISA. Il a récemment publié

- Odonne G., Molino J.-F., 2018. Écologie historique amazonienne, une interdisciplinarité nécessaire quand l'archéologue est perdu dans les bois... Les nouvelles de l'archéologie, $\mathrm{n}^{\circ} 152$, p. 11-15.

- Ogeron C., Odonne G. et al., 2018. Palikur traditional roundwood construction in Eastern French Guiana: ethnobotanical and cultural perspectives. Journal of Ethnobiology and Ethnomedicine [En ligne]. https://ethnobiomed.biomedcentral.com/articles/10.1186/s13002-018-0226-7

- Tareau, M.-A., Palisse, M., Odonne, G., 2017. As vivid as a weed... Medicinal and cosmetic plant uses amongst the urban youth in French Guiana. Journal of Ethnopharmacology, n² 203, p. 200-213.

\section{MARIANNE PALISSE}

Marianne Palisse, marianne.palisse@univ-guyane.fr, maître de conférences en anthropologie, Université de Guyane, LEEISA, USR 3456. Elle a récemment publié :

- Tareau, M.-A., Palisse, M., Odonne, G., 2017. As vivid as a weed... Medicinal and cosmetic plant uses amongst the urban youth in French Guiana. Journal of Ethnopharmacology 203: 200-213.

- Palisse, Marianne, 2016. Les pratiques agricoles des migrants haïtiens en Guyane : entre insertion et stigmatisation. In Collomb G., Mam Lam Fouck S., Mobilités, ethnicités et diversité culturelle. La Guyane entre Surinam et Brésil, Ibis Rouge, pp. 189-206.

- Palisse, Marianne, 2014. La Faculté d'Ethnologie de l'Université d'État d'Haïti : des projets de Price-Mars et de Roumain à la réalité des années 2000. In Byron J.P. (dir.), Production du savoir et construction sociale. L'ethnologie en Haïti, Presses de l'Université de Laval / Éditions de l'Université d'État d'Haïti, pp. 83-98.

\section{CLARISSE ANSOE}

Clarisse Ansoe, ansoe.clarissemana@gmail.com, étudiante en Lettres à l'Université de Guyane et interprète en langue nenge tongo. 